\title{
EELS Investigation of the Formulas for Inelastic Mean Free Path
}

\author{
Huairuo Zhang, ${ }^{*}$ Ray F. Egerton ${ }^{* * *}$ and Marek Malac ${ }^{*, * *}$ \\ *National Institute for Nanotechnology, Edmonton, Canada, T6G 2M9 \\ ${ }^{* *}$ Department of Physics, University of Alberta, Edmonton, Canada, T6G 2G7
}

Electron energy-loss spectroscopy (EELS) is routinely used to estimate local specimen thickness in a TEM. The relative specimen thickness is conveniently estimated from the low-loss spectrum using log-ratio method with a formula $t / \lambda=$ $\ln \left(I_{t} / I_{0}\right)$ [1]. Here $\lambda$ is an inelastic mean free path (IMFP) for the material in question, $I_{0}$ is the area under zero-loss peak (ZLP) and $I_{\mathrm{t}}$ the total area under the whole spectrum. An absolute thickness $t$ measurement requires knowledge of the IMFP, which depends on the specimen material, electron energy and collection semiangle. The most commonly used IMFP formula, incorporated into Gatan DigitalMicrograph software, was developed by Malis et al. and exhibits a smooth dependence on atomic number $Z[2]$ :

$\lambda=106 F E_{0} /\left[E_{m} \ln \left(2 \beta E_{0} / E_{m}\right)\right]$

with $E_{\mathrm{m}}=7.6 Z^{0.36}$. The Malis formula is valid only within the dipole region of scattering (collection semiangles $\beta<20 \mathrm{mrad}$ ). More recently, Iakoubovskii et al. developed a new formula in which IMFP is parameterized in terms of specimen density $\rho$, making allowance for the convergence angle $\alpha$ of the electron probe [3]:

$\lambda=200 F E_{0} /\left\{11 \rho^{0.3} \ln \left[\frac{\alpha^{2}+\beta^{2}+2 \theta_{E}^{2}+\left|\alpha^{2}-\beta^{2}\right|}{\alpha^{2}+\beta^{2}+2 \theta_{C}^{2}+\left|\alpha^{2}-\beta^{2}\right|} \times \frac{\theta_{C}^{2}}{\theta_{E}^{2}}\right]\right\}$

Figure 1 plots the calculated IMFP values of thirteen elemental materials, for collection semiangle $2.6 \mathrm{mrad}$, accelerating voltage $100-300 \mathrm{kV}$, assuming bulkmaterial densities. Unlike the smoothly decreasing IMFP given by the Malis formula, the IMFP values of Iakoubovskii show a pronounced fluctuation with increasing atomic number and are generally larger than Malis values. As shown in Fig. 2, the discrepancy increases with increasing accelerating voltage and increasing of atomic number. A well-defined $\mathrm{MgO}$ nanocube of $100 \mathrm{~nm}$ thickness was used to assess the accuracy of these two formulas. Figure 3 shows the ratio of measured thickness using the two IMFP formulas to the actual $\mathrm{MgO}$ thickness, as a function of accelerating voltage and collection semiangle. The measurement on $\mathrm{MgO}$ nanocube suggests that the Malis method is preferable at small collection semiangles, while the Iakoubovskii method is preferable for medium and large collection semiangles.

[1] R.F. Egerton. Electron Energy-Loss Spectroscopy in the Electron Microscope, 3rd ed, Springer, New York, 2011.

[2] T. Malis, S.C. Cheng, R.F. Egerton. J. Electron Microsc. Tech. 8 (1988) 193-200.

[3] K. Iakoubovskii et al. Res. Tech. 71 (2008) 626-631.

[4] Financial support from NINT and NSERC is gratefully acknowledged. 


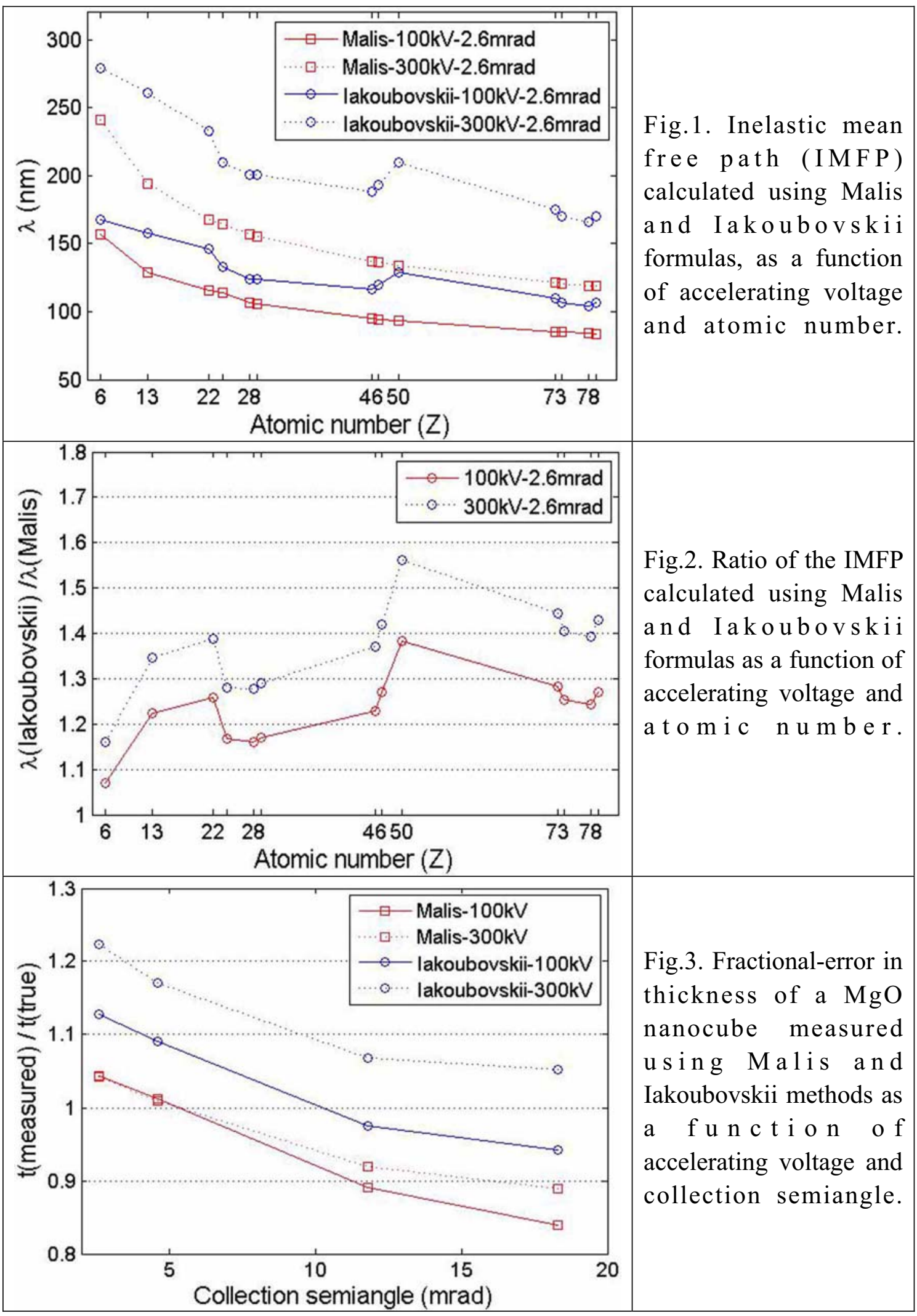

